\title{
Shear Alignment Behavior of Nematic Solutions Induced by Ultralong Side-Group Liquid Crystal Polymers
}

\author{
M. D. Kempe and J. A. Kornfield \\ California Institute of Technology, 1200 East California Boulevard, Pasadena, California 91125
}

(Received 10 October 2002; published 17 March 2003)

\begin{abstract}
Addition of a low concentration of a very long $(430 \mathrm{~kg} / \mathrm{mol})$ side group liquid crystal polymer is shown to produce dramatic changes in the flow characteristics of a calamitic nematic liquid crystal. This polymer causes a typical flow-aligning nematic liquid crystal to align near the velocity gradient direction rather than near the velocity direction, corresponding to having a tumbling parameter $\lambda<-1$, for concentrations greater than $7.5 \%$ polymer. Such flow-aligning behavior has not been reported previously in a calamitic nematic. The large molecular weight of the present polymer relative to those examined in the prior literature is responsible for these new phenomena.
\end{abstract}

DOI: 10.1103/PhysRevLett.90.115501

The effects of polymeric materials on the orientation and dynamics of liquid crystals (LCs) are used to enhance the performance and fabrication of electro-optic devices by conferring the desired alignment, mechanical stability, or viscosity. Polymers are used in conjunction with LCs as alignment layers, polymer dispersed LCs $[1,2]$, polymer stabilized LCs [3,4], polymer LC gels $[5,6]$, and polymers dissolved in LC hosts [7,8]. Therefore, the coupling of orientation and dynamics between polymers and liquid crystals is of great interest. Here we focus on polymers that are dissolved in an LC host and probe the coupling between them by examining the orientational response during shear.

In nematic LCs the orientational distribution of the molecules is uniaxial with a preferred orientation called the "director." The flow behavior of nematics is rich due to the transient response of the director. For example, if initially aligned along the gradient direction, inception of shear causes the director of all known calamitic (i.e., rodshaped as opposed to disk-shaped, "discotics") nematic liquid crystals to rotate in the same direction as the vorticity, analogous to rodlike particles in suspension. However, the constraints of thermodynamics and the symmetry of the fluid admit the possibility that the nematodynamic torque could rotate the director in the opposite direction. Here we provide the first report of such behavior.

Two classes of behavior, flow-aligning and tumbling, of the nematic director occur under the influence of shear. Under deformations fast enough that viscous effects dominate over elastic effects, Ericksen's transversely isotropic fluid (TIF) model [9] can describe the evolution of the director

$$
\frac{D \vec{n}}{D t}=\vec{n} \cdot \underline{\underline{\omega}}+\lambda(\vec{n} \cdot \underline{\underline{d}}-\vec{n} \vec{n} \vec{n}: \underline{\underline{d}}) .
$$

Here $\underline{\underline{d}}$ is the symmetric part of the velocity gradient tensor and $\underline{\underline{\omega}}$ is the vorticity tensor. The tumbling parameter, $\lambda$, of a given LC determines its response to an
PACS numbers: 61.30.-v, 61.30.Cz, 61.30.Dk, 61.30.Vx

imposed strain. For values of $|\lambda|>1$ the LC is flow aligning and will rotate under shear to a steady state angle determined by $\lambda$. But when $|\lambda|<1$ the LC is a tumbling nematic and the director continuously rotates in response to a shear strain.

Calamitic LCs typically have a tumbling parameter slightly greater than 1 [10] and adopt a steady state angle close to the velocity direction. Occasionally, a calamitic LC will have a tumbling parameter between 0 and 1 , usually associated with a nematic LC at a temperature near a smectic transition [11]. On the other hand, nematic discotic LCs are predicted to have tumbling parameters less than -1 [12], in accord with experiment [13]. Under a steady shear stress, the discotic director will align nearly parallel to the velocity gradient direction enabling the platelike molecules to slide over each other.

Dissolving a polymer with an anisotropic conformation in a small-molecule LC host can affect the tumbling parameter. The rheological theory of Brochard [14] predicts changes in the viscosity coefficients as functions of the polymer anisotropy, concentration, and relaxation time. In particular, this model predicts that oblate polymers $\left(R_{\|}<R_{\perp}\right)$ will reduce $\lambda$ while prolate polymers will increase $\lambda$. These general predictions have been borne out: oblate side group liquid crystal polymers (SGLCPs) have been shown to convert a flow-aligning host $(\lambda>1)$ to a tumbling type, and main-chain liquid crystal polymers can convert a tumbling host $(0.1<\lambda<1)$ to flow aligning $(\lambda>1)$ [15].

Furthermore, Brochard predicts that if enough oblate polymer is added to a calamitic LC, the tumbling parameter can be made to fall below -1 , resulting in alignment near the velocity gradient direction [16]. Despite more than two decades of research, this prediction has not been validated prior to this work. Values of $\lambda$ only as low as $\sim 0.7$ [17] have been reached for a flowaligning host [18]. Only in the high concentration limit of a polymer melt has $\lambda<0$ been observed [19]. Strikingly, prior investigations have been limited to relatively short 
polymers (degree of polymerization D.P. $\leq 100$ ) [20]. By examining much longer chains (D.P. $\sim 1200)$ with comparable anisotropy to previous systems $\left(R_{\perp} / R_{\|} \approx 1.5\right)$ [21] much more dramatic effects are observed. In particular, we show that at modest concentration $(>7.5 \% \mathrm{wt})$ a conventional flow-aligning host $(\lambda>1)$ can be converted to one that is flow aligning with $\lambda<-1$.

The polymer used in our study was synthesized with a 1,2-polybutadiene backbone by attaching a cyanobiphenyl based mesogen to each pendant vinyl group, Fig. 1 [22]. The details of the synthesis and characterization of this polymer will be published elsewhere [23]. Starting with an anionically produced 1,2-polybutadiene backbone of $65000 \mathrm{~g} / \mathrm{mol}$, a final polymer of $430000 \mathrm{~g} / \mathrm{mol}$ was obtained with a low polydispersity $\left(M_{w} / M_{n} \cong 1.15\right)$. This polymer was dissolved in the nematic solvent 4'-pentyl-4-biphenylcarbonitrile (5CB) at concentrations of $0.5 \%$ to $10 \%$ by weight. To do this, the polymer and LC were dissolved in dichloromethane and placed under vacuum at room temperature for two days to remove the dichloromethane, leaving behind the polymer solutions in 5CB.

Experimental determination of $\lambda$ is based on a 2D version of Erickson's TIF model describing director motion that remains in the plane defined by the velocity and the velocity gradient directions [24]. This approximation is valid when the Ericksen number, $E r=$ $h^{2} \dot{\gamma}\left(\alpha_{3}-\alpha_{2}\right) / K_{1}$, which is the ratio of the viscous to elastic torques, is much greater than one. When $E r \gg 1$, rheological transients in nematics are predicted to scale with strain. To confirm the regime in which $E r \gg 1$, experiments with $5 \mathrm{CB}$ were performed at different strain rates and/or sample thicknesses. Typically [25], addition of polymer increases viscosity while leaving elasticity relatively unchanged, thereby increasing $E r$; therefore, shear rates that correspond to $E r \gg 1$ for $5 \mathrm{CB}$ all lie in the high $\mathrm{Er}$ regime for all our polymer solutions as well.

For director rotation the 2D TIF model yields

$$
\frac{\partial \theta}{\partial \gamma}=\frac{\left(\alpha_{3} \sin ^{2} \theta-\alpha_{2} \cos ^{2} \theta\right)}{\alpha_{3}-\alpha_{2}},
$$

with the transient stress described in terms of an apparent viscosity given by

$$
\begin{aligned}
\eta_{\text {app }} \equiv \frac{\tau_{x y}}{\dot{\gamma}}= & {\left[\alpha_{1}+\frac{\left(\alpha_{2}+\alpha_{3}\right)^{2}}{\alpha_{3}-\alpha_{2}}\right] \sin ^{2} \theta \cos ^{2} \theta } \\
& +\frac{1}{2}\left(\alpha_{3}+\alpha_{4}+\alpha_{6}\right)-\frac{\alpha_{3}^{2}}{\alpha_{3}-\alpha_{2}} .
\end{aligned}
$$

Here $\theta$ is the angle of the director measured relative to the

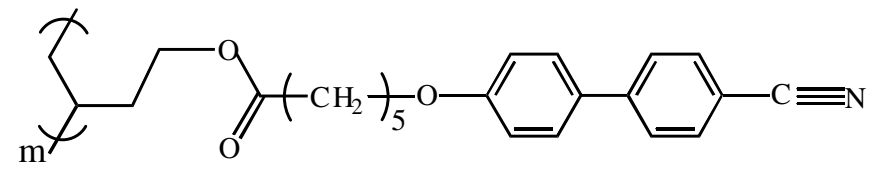

FIG. 1. Side-group liquid crystal polymer PBCB6. velocity gradient, $\gamma$ is the strain, and the $\alpha$ 's are the Leslie-Erickson viscous coefficients. Using Eq. (2) for small strains, measurement of $\theta(\gamma)$ can be used to determine the tumbling parameter

$$
\lambda=\frac{\alpha_{2}+\alpha_{3}}{\alpha_{2}-\alpha_{3}}=2 \frac{\theta(\gamma)}{\gamma}-1
$$

If the LC is of the flow-aligning type, then the magnitude of the steady state angle, $\theta_{s s}$, of the director under shear can also be used:

$$
\lambda=-\frac{1}{\cos \left(2 \theta_{s s}\right)} .
$$

For a tumbling nematic, integration of Eq. (2) yields

$$
\tan (\theta)=\left(\frac{1+\lambda}{1-\lambda}\right)^{1 / 2} \tan \left(\frac{\gamma}{2} \sqrt{1-\lambda^{2}}\right) .
$$

From this, the period of the stress oscillations corresponding to a director rotation of $180^{\circ}, \gamma_{p}$, can be used to determine the tumbling parameter using

$$
\lambda= \pm \sqrt{1-\frac{4 \pi^{2}}{\gamma_{p}^{2}}} .
$$

Here one must look at the transient stress response to determine the sign of $\lambda$. By inspection of Eq. (3) one can see that stress maxima occur when the director is at an angle of $\pm 45^{\circ}$ and that minima occur at $0^{\circ}$ or $90^{\circ}$. Furthermore, Eq. (2) indicates that the relative rate of change of $\theta$ with strain is determined by $\alpha_{2}$ or $\alpha_{3}$ when the director is at $0^{\circ}$ or $90^{\circ}$, respectively. Therefore, if $\left|\alpha_{2}\right|>\left|\alpha_{3}\right|$ the director will rotate faster at $0^{\circ}$ than it will at $90^{\circ}$ indicating a positive tumbling parameter or vice versa.

For the case where $|\lambda|>1$ (flow-aligning), integration of Eq. (2) yields

$$
\tan (\theta)= \pm\left(\frac{1+\lambda}{\lambda-1}\right)^{1 / 2} \tanh \left(\frac{\gamma}{2} \sqrt{\lambda^{2}-1}\right) .
$$

Using this expression for $\theta(\gamma)$, one can fit Eq. (3) to the rheological response and obtain a value for $\lambda$. Furthermore, inspection of this equation shows that the two different cases of flow-aligning nematics are readily distinguished in their transient rheological response during inception of steady shear from an initially homeotropic monodomain $\left(\theta=0^{\circ}\right)$. For $\lambda>1$, the steady state angle is in the range $45^{\circ}<\theta_{s s}<90^{\circ}$, and a stress overshoot occurs as $\theta$ rotates through $+45^{\circ}$. On the other hand, for $\lambda<-1, \theta_{s s}$ lies between $-45^{\circ}$ and $0^{\circ}$, so the stress monotonically increases as the director rotates from $0^{\circ}$ to $\theta_{s s}$.

Here we use measurements of both $\theta(\gamma)$ and $\tau_{x y}(\gamma)$ to obtain two independent determinations of $\lambda$. In both conoscopy $[18,24,26]$ and rheology experiments, the sample was placed between lecithin treated surfaces to promote perpendicular orientation of the director relative to the surfaces. For conoscopy, samples were placed 
between two parallel glass plates separated by 0.25 to $0.50 \mathrm{~mm}$ and viewed between crossed polarizers using a highly convergent beam of light. The observed interference figure is used to determine the angle of the director relative to the velocity gradient direction, $\theta$ [27]. The value of $\lambda$ is determined from $\theta(\gamma)$ at small $\theta$ using Eq. (4) (Fig. 2). For samples that are flow aligning, the steady state angle provides an alternate determination of $\lambda$ [Eq. (5)]. In practice, the center of the interference figure remains in the field of view at steady state only for samples with $\lambda<-1$; in this case the values of $\lambda$ determined by Eqs. (4) and (5) gave consistent results that differ only by 0.04 .

For the rheological determination of $\lambda$, a rheometrics fluids rheometer was used with a $50 \mathrm{~mm}$ diameter cone and plate having a cone angle of $0.02 \mathrm{rad}$. The apparent viscosity was recorded as a function of strain during inception of steady shear. For the tumbling case the strain period, $\gamma_{p}$, was used in Eq. (7) to determine $|\lambda|$. The sign of $\lambda$ was determined by inspection of $\tau_{x y}(\gamma)$. Since our experiments were conducted with the director initially in the perpendicular position $\left(\theta=0^{\circ}\right)$, the presence of a single stress maximum followed by two stress maxima closer together, e.g., Fig. 3(b), results from faster director movement at $0^{\circ}$ than $90^{\circ}$. This indicates that $\left|\alpha_{2}\right|>\left|\alpha_{3}\right|$ and that the tumbling parameter is positive. Conversely, observing the first two peaks closer together indicates a negative tumbling parameter, e.g., Fig. 3(c).

At all concentrations of polymer, measurements of the tumbling parameter using both conoscopy and rheology gave consistent results. At $7.5 \mathrm{wt} \%$ polymer, even under shear strains as high as 20 , the conoscopic image barely moves $(\lambda \approx-1)$. This is in sharp contrast to pure $5 \mathrm{CB}$, for which the center of the conoscopic figure moves out of view after only 0.14 strain units. While performing conoscopic measurements with $7.5 \%$ polymer at temperatures from 20 to $33{ }^{\circ} \mathrm{C}$ it was noted that the direction of deflection of the conoscopic image changed sign at $24^{\circ} \mathrm{C}$ indicating that $\alpha_{2} \cong 0$ and $\lambda \cong-1$. This value for $\lambda$ produces a nematic solution that, in contrast to typical calamitic LCs, will align nearly parallel to the velocity gradient direction rather than the flow direction. To our knowledge, this is the first report of $\lambda \cong-1$ for a calamitic nematic system.

At $10 \mathrm{wt} \%$ polymer, the solution is distinctly flow aligning with $\lambda<-1$, Fig. 3(d). Conoscopic observation

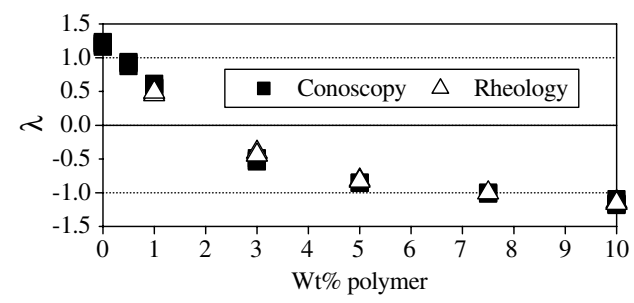

FIG. 2. Rheological and conoscopic measurements of the tumbling parameter for PBCB6 dissolved in $5 \mathrm{CB}$ at $25^{\circ} \mathrm{C}$. of $\theta(\gamma)$ gives $\lambda=-1.19$ based on the small strain response, Eq. (4). Under large strains a steady state alignment angle of $\theta_{s s}=-15^{\circ}$ gives $\lambda=-1.15$, Eq. (5). The stress response also indicates that the director never goes through an angle of $\pm 45^{\circ}$ which would produce a stress overshoot as is seen for flow alignment with $\lambda>1$ [e.g., pure 5CB, Fig. 3(a) [18]]. Instead, a steady stress is monotonically approached with $\tau_{x y}(\gamma)$ corresponding to $\lambda=-1.13$.

To estimate the polymer relaxation time, the dynamic rheological response of the LC solutions was also characterized. For all polymer concentrations examined, only the terminal region was accessible. For the $10 \%$ solution at $25^{\circ} \mathrm{C}$, it was possible to estimate the crossover frequency, $G^{\prime}=G^{\prime \prime}$, by extrapolating using a slope of two and one for $G^{\prime}$ and $G^{\prime \prime}$, respectively. The intercept of these two lines was at a frequency of $\omega=1770 \mathrm{rad} / \mathrm{s}$. This frequency corresponds to a strain rate of $280 \mathrm{~s}^{-1}$ which is more than an order of magnitude greater than the strain rates of 4 to $32 \mathrm{~s}^{-1}$ that were used in the transient rheology experiments. This indicates that the new phenomena seen in these experiments are not due to significant stretching of the polymer.
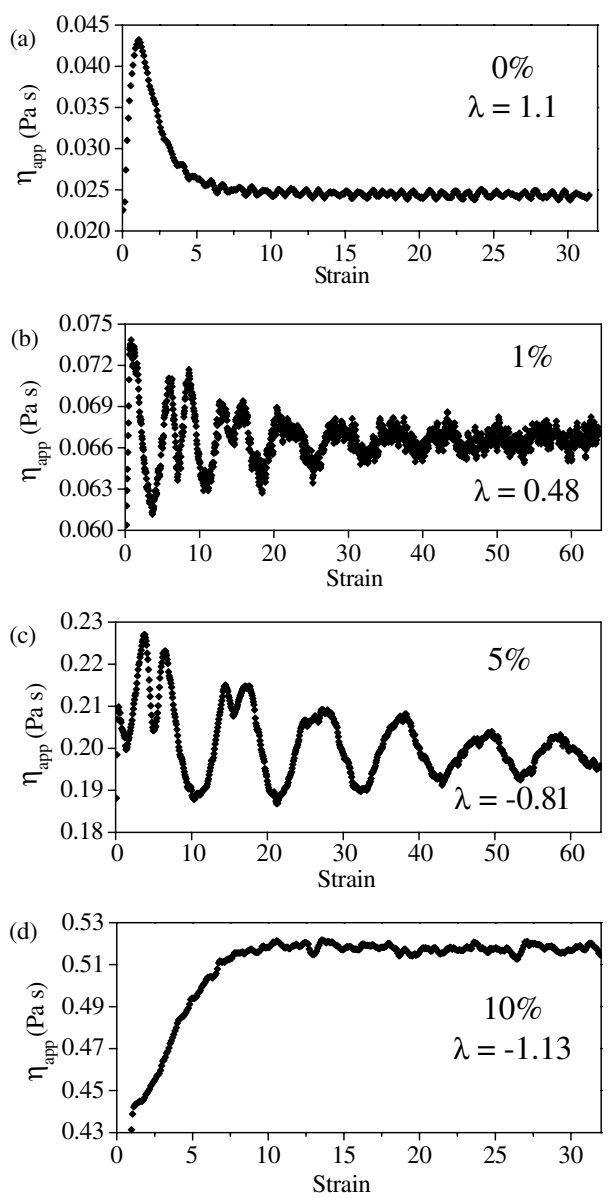

FIG. 3. Transient response of (a) pure 5CB and solutions containing (b) $1 \%$, (c) 5\%, and (d) $10 \%$ PBCB6. Strain rate was $16 \mathrm{~s}^{-1}$ at $25^{\circ} \mathrm{C}$. 

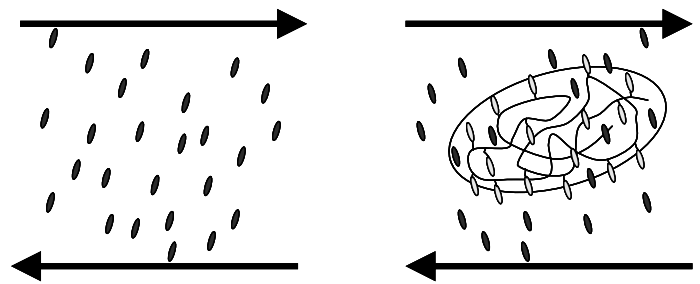

FIG. 4. Schematic diagram showing how the presence of an oblate polymer can create a negative tumbling parameter and cause the director to rotate counter to the vorticity.

The appearance of a flow-aligning condition near the velocity gradient direction can be explained as resulting from the competition between two opposing torques on the director, Fig. 4. A shearing force applied to an oblate polymer would tend to orient its long axis along the extensional axis of shear $\left(+45^{\circ}\right)$. Since in the oblate polymer the mesogen is perpendicular to the backbone, the pendant mesogens will exert a torque on the director rotating it toward an angle of $-45^{\circ}$; from an initial orientation $\theta=0^{\circ}$, this rotates $\boldsymbol{n}$ against the vorticity. This contrasts the LC host, $5 \mathrm{CB}$, which under shear starting from $\theta=0^{\circ}$ experiences a torque on the director rotating it with the vorticity toward the velocity direction. The competition between these two torques leads to flow alignment near the velocity gradient direction with $\lambda<-1$.

In order for the tumbling parameter to be less than -1 and for the director to rotate counter to the vorticity when $\theta=0^{\circ}, \alpha_{2}$ must be positive. This is in qualitative agreement with Brochard theory. To date, the only other measurements of negative tumbling parameters has been in discotic [13] systems and with melt SGLCPs [19]. By using polymers with a molecular weight that is typically at least an order of magnitude larger than other researchers [20], we were able to get much greater changes in viscoelastic properties using less polymer. Not only does this study open up new regimes of the tumbling parameter for study, but it also verifies some aspects of Brochard theory. She predicted that the addition of an oblate polymer to a flow-aligning LC can cause the tumbling parameter to be reduced below -1 to produce flow alignment near $\nabla \vec{\nu}$ instead of $\vec{\nu}$ as is seen in typical calamitic LCs.

We thank Chris Ober and Jagdish Jethalamari for help with synthesis, Weijun Zhou for help with instrumentation, and AFOSR LC-MURI for funding for this project.

[1] R. L. Sutherland, V. P. Tondiglia, L. V. Natarajan, T. J. Bunning, and W.W. Adams, Appl. Phys. Lett. 64, 1074 (1994).

[2] H. S. Kitzerow, Liq. Cryst. 16, 1 (1994).

[3] H. Furue, H. Yokoyama, and S. Kobayashi, Jpn. J. Appl. Phys. 40, 5790 (2001).

[4] K. Kang, L. C. Chien, and S. Sprunt, Liq. Cryst. 29, 9 (2002).
[5] C. C. Chang, L. C. Chien, and R. B. Meyer, Phys. Rev. E 56, 595 (1997).

[6] R. A. M. Hikmet, H. M. J. Boots, and M. Michielsen, J. Appl. Phys. 79, 8098 (1996).

[7] H. J. Coles and M. S. Bancroft, Mol. Cryst. Liq. Cryst. 237, 97 (1993).

[8] M. C. Chang, H.W. Chiu, X. Y. Wang, T. Kyu, N. Leroux, S. Campbell, and L. C. Chien, Liq. Cryst. 25, 733 (1998).

[9] J. L. Ericksen, Arch. Ration. Mech. Anal. 4, 231 (1960); F. M. Leslie, ibid. 28, 265 (1968).

[10] D. F. Gu and A. M. Jamieson, J. Rheol. 38, 555 (1994).

[11] P. Pieranski and E. Guyon, Phys. Rev. Lett. 32, 924 (1974); C. Gahwiller, ibid. 28, 1554 (1972); P. E. Cladis and S. Torza, ibid. 35, 1283 (1975).

[12] T. Carlsson, Mol. Cryst. Liq. Cryst. 89, 57 (1982); G. E. Volovik, JETP Lett. 31, 273 (1980).

[13] J. T. Mang, S. Kumar, and B. Hammouda, Mol. Cryst. Liq. Cryst. 303, 244 (1997); A. B. D. Brown and A. R. Rennie, Chem. Eng. Sci. 56, 2999 (2001).

[14] F. Brochard, J. Polym. Sci., Polym. Phys. Ed. 17, 1367 (1979).

[15] D. F. Gu and A. M. Jamieson, Macromolecules 27, 337 (1994).

[16] D. F. Gu, A. M. Jamieson, and S. Q. Wang, J. Rheol. 37, 985 (1993).

[17] N. Yao and A. M. Jamieson, Macromolecules 31, 5399 (1998).

[18] Even though tumbling nematic LCs can have tumbling parameters as low as $\lambda \sim 0.2$, only $\lambda \sim 0.4$ has been reported for a solution of a polymer dissolved in a tumbling nematic. D. J. Ternet, R. G. Larson, and L. G. Leal, Rheol. Acta 38, 183 (1999); N. Yao and A. M. Jamieson, Macromolecules 31, 5399 (1998).

[19] I. Quijada-Garrido, H. Siebert, P. Becker, C. Friedrich, and C. Schmidt, Rheol. Acta 38, 495 (1999); I. QuijadaGarrido, H. Siebert, C. Friedrich, and C. Schmidt, Macromolecules 33, 3844 (2000); H. Siebert, P. Becker, I. Quijada-Garrido, D. A. Grabowski, and C. Schmidt, Solid State Nucl. Magn. Reson. 22, 311 (2002).

[20] A. M. Jamieson, D. Gu, F. L. Chen, and S. Smith, Prog. Polym. Sci. 21, 981 (1996).

[21] Preliminary results of SANS data on solutions of SGLCP in deuterated $5 \mathrm{CB}$ indicate a polymer anisotropy of about $R_{\perp} / R_{\|}=1.5$ and that the overlap concentration is around $20 \%$. This anisotropy is similar to SANS studies on SGLCPs in the melt. Brochard theory predicts that greater polymer anisotropy will have a greater effect on the tumbling parameter.

[22] T. C. Chung, M. Raate, E. Berluche, and D. N. Schulz, Macromolecules 21, 1903 (1988); J. Sanger, W. Gronski, S. Maas, B. Stuhn, and B. Heck, ibid. 30, 6783 (1997).

[23] M. D. Kempe and J. A. Kornfield (to be published).

[24] P. T. Mather, D. S. Pearson, and W. R. Burghardt, J. Rheol. 39, 627 (1995).

[25] P.Y. Liu, N. Yao, and A. M. Jamieson, Macromolecules 32, 6587 (1999).

[26] W. Zhou, J. A. Kornfield, and W. R. Burghardt, Macromolecules 34, 3654 (2001).

[27] F. D. Bloss, An Introduction to the Methods of Optical Crystallography (Holt, Rinehart \& Winston, New York, 1961). 